\title{
Omics of Root-to-Shoot Signaling Under Salt Stress and Water Deficit
}

\author{
Francisco Pérez-Alfocea, ${ }^{1}$ Michel Edmond Ghanem, ${ }^{1}$ Aurelio Gómez-Cadenas, ${ }^{2}$ and lan C. Dodd ${ }^{3}$
}

\begin{abstract}
Maximizing crop yield depends on the leaves receiving an optimal supply of water, mineral nutrients, small organic molecules, proteins, and hormones from the root system via the xylem. Soil drying and salinization alter these xylem fluxes, and modern omics techniques offer unparalleled opportunities to understand the complexity of these responses. Although absolute xylem concentrations of any constituent depend on the genotype and xylem sap sampling methodology, analysis of the relative changes in concentrations has revealed some conserved behavior. Typically, these stresses increase xylem concentrations of the plant hormone abscisic acid (ABA) that limits crop water loss, but decrease the concentrations of certain cytokinins that stimulate expansive growth and prevent premature leaf senescence. Further understanding of the ionic and biophysical alterations in the rhizosphere environment that cause increased xylem concentrations of the ethylene precursor (ACC) is needed. Interactions of these plant hormones with plant nutrient status and xylem nutrient delivery may be important in tuning plant responses to their environment. Xylem proteomics is an emerging area that will help understand mechanisms of plant stress adaptation. Using omics techniques to underpin rootstock-mediate plant improvement is likely to improve crop yields in dry or saline soil.
\end{abstract}

\section{Introduction}

G LOBAL FOOD SECURITY for an expanding world population is increasingly threatened by environmental stresses that limit the growth and yield of our major crop species. Soil water deficits and salinization are the two most intractable abiotic stresses that limit the production of the world's food crops (Munns, 2011) thus maintaining crop productivity even under unfavorable environments is a major area of research in the plant sciences. Developing crop plants that can grow and yield better under salt and water stress conditions, demands novel strategies and relies on the use of innovative technologies to comprehensively understand plant responses to abiotic stresses.

Plant productivity depends on photosynthetic processes in the leaves that are strongly dependent on an adequate supply of water, mineral nutrients, small organic molecules, proteins, and hormones. Most of these are either synthesized in (i.e., hormones, amino acids, proteins) or taken up (i.e., water and mineral nutrients) by the roots and have to be transported to the shoot to enable normal leaf functioning. In compensation, a flux of assimilates from shoot to roots assures normal root functioning and continued resource uptake by the roots. The composition of the xylem (xylomics) is important not only to provide the shoot with optimal nutrition, but also to regulate the necessary adaptive responses in the different plant organs (leaves, roots, and vascular system) to optimize resource acquisition under unfavorable conditions. Root or shootderived changes in these chemical signals in the xylem usually act to enhance plant adaptation to harmful conditions (i.e., reducing the water loss) while other stress-induced changes directly affect the survival of plant (e.g., premature leaf senescence). These changes lessen plant productivity under suboptimal conditions. The identification and understanding of those chemical changes in the roots and their specific effects in the shoot tissues could open new strategies to improve crop yield stability under abiotic stress conditions such as water deficit and saline stress.

This minireview considers innovative "omics" technologies that have evolved during the last few decades to study plant abiotic stress responses. Understanding (root or shoot) tissue responses requires the measurement of changes in gene expression (termed genomics or transcriptomics). However, because studying root-to-shoot transport processes primarily seeks to determine xylem sap composition, this review largely considers the metabolomics, ionomics, proteomics

\footnotetext{
${ }^{1}$ Plant Nutrition, CEBAS-CSIC, Campus de Espinardo, Espinardo, Murcia, Spain.

${ }^{2}$ Ciències Agràries i del Medi Natural, Universidad Jaume I, , Castellon de la Plana, Spain.

${ }^{3}$ The Lancaster Environment Centre, Lancaster University, Lancaster, United Kingdom.
} 
and "hormone-omics" of this transport fluid. Collecting this transport fluid is not without methodological difficulties, and a comprehensive review of available techniques and their relative advantages has been published (Dodd, 2005). Detailed analysis of xylem sap composition in relation to salt and water stresses can provide information about the structure and complexity of the physiological mechanisms that are correlated with specific ionic (Albacete et al., 2009) and biophysical stresses (e.g., Dodd et al., 2010) in the rhizosphere, and reveal unexpected or previously uncharacterized biochemical interactions. Xylem sap profiling is likely to provide physiological markers to assist root-targeted breeding for resistance to individual and combined abiotic stresses.

\section{Metabolomics of Environmental Stress}

The final goal of a metabolomics analysis is to identify and quantify all metabolites in a given organism and assess the metabolic interrelationships among them. To date, this has not been possible because most metabolites are still unknown and the available analytical techniques do not allow such exhaustive metabolite detection (Enot et al., 2000). Therefore, the most widely used techniques, known as metabolite profiling (Fiehn et al., 2000), analyze a restricted number of metabolites in a given sample. Mass spectrometry (MS) coupled to separative techniques such as high-performance liquid chromatography (HPLC), gas chromatography (GC), or capillary electrophoresis are most often used for this purpose (De Vos et al., 2007).

Metabolomics can contribute significantly to the study of stress metabolism, stress signal transduction molecules, or molecules that are part of the acclimation response of plants (Ruan et al., 2010). Most of the mechanisms activated in plants by environmental stresses aim to reestablish cellular biochemical homeostasis, although this is rarely achieved (Bohnert el al., 2006). There have been some agronomic successes in manipulating gene expression of key components of stress tolerance (Bohnert el al., 2006), but limited knowledge of stress-associated metabolism remains a major gap to understanding the mechanisms of plant tolerance to abiotic stress.

Within this context, there has been relatively little work in analysing xylem sap metabolite profiles under different environmental stresses. Amino acid concentration and composition in xylem sap and in plant tissues was determined to be good markers of plant performance in Eucalyptus regnans grown under different environmental conditions (Pfautsch et al., 2009). In Brassica oleracea, the xylem sap metabolome comprised many organic compounds such as sugars, organic acids, and amino acids. Of these, salt stress ( $80 \mathrm{mM}$ for 7 days) substantially reduced amino acid concentrations, especially glutamine, the major xylem sap amino acid (FernandezGarcía et al., 2011). However, total and individual (e.g., asparagine, glutamine, proline, and aspartic, glutamic, and $\gamma$ aminobutyric acids) amino acids and soluble sugars concentration increased by twofold in the xylem of tomato plants exposed for 3 weeks to $100 \mathrm{mM} \mathrm{NaCl}$ salt stress (MartínezAndujar, 2006; Pérez-Alfocea et al., 2000). Because increases in amino acid concentration in the xylem sap seem to occur from the first day of salinization in tomato (Pérez-Alfocea et al., 2000), it cannot be excluded that this response to the stress depends on the plant species and/or the constitutive major Nforms transported in the xylem (e.g., nitrate vs. amino acids).
In rice (Oryza sativa), although the xylem sap metabolome was not analyzed, salt stress strongly decreased concentrations of tri-carboxylic acid cycle intermediates, pyruvic, citric, aconitic, malic, and 2-oxoglutaric acids, concomitant with reductions in the levels of shikimic and quinic acids and increases in amino acids (Zhuter et al., 2007). However, the plant response and, therefore, the xylem composition of these organic molecules seem to depend on the light conditions. Indeed, decreased organic acid concentrations were observed in leaves and roots of salinized $(100 \mathrm{mM} \mathrm{NaCl} \times 3$ weeks $)$ tomatoes harvested in the dark period, whereas these were not affected in the xylem sap of plants harvested during the light period (Martínez-Andujar, 2006). Nevertheless, although the xylem sap was not analyzed, the concentration of tri-carboxylic acid cycle intermediates in the leaves harvested during the dark period increased by twofold under salinity compared to control plants (Martinez-Andujar, 2006).

Changes in the plant metabolome in response to drought stress seem to depend on prevailing environmental conditions (e.g., atmospheric temperature, relative humidity). Levels of a number of metabolites (proline, valine, threonine, homoserine, myoinositol, gamma-aminobutyrate, and nicotinic acid) always increased with drought stress in pea (Pisum sativum) plants under different growing conditions (Charlton et al., 2008), but a set of metabolites that were altered in relative amounts in different experiments, but not specifically associated with drought stress, was also identified, including glutamate, asparagine, and malate.

Other authors working with Arabidopsis thaliana have found that short-term responses to salt stress included the induction of the methylation cycle for the supply of methyl groups, the phenylpropanoid pathway for lignin production and glycine betaine production. Long-term effects included the coinduction of glycolysis and sucrose metabolism and coreduction of the methylation cycle (Kim et al., 2007). Studying the metabolism of halophytes can improve our understanding of cellular responses to ion intoxication. Authors working with different species have reached common conclusions suggesting that after stress imposition, the physiological responses in non-adapted species are induced through hormonal regulation, whereas better adapted genotypes present a basal metabolic configuration better able to endure environmental cues (Arbona et al., 2010; Gagneul et al., 2007; Gong et al., 2005).

\section{Xylem Hormonomics Under Water and Saline Stress}

Salinity and drought affect plant productivity by reducing the photosynthetic area by inhibiting cell division and cell expansion rates during leaf growth and by affecting developmental programs regulating leaf emergence, the production of lateral primordia, and the formation of reproductive organs (summarized in Munns, 2002). Whether water status, hormonal regulation, or supply of photosynthate exerts dominant control over growth of plants in dry or saline soil is an issue that has been hotly debated. Over the time scale of days, there is much evidence to suggest that hormonal signals rather than water relations are controlling growth in saline soils (Munns, 2011; Pérez-Alfocea et al., 2010) because leaf expansion in saline soil only transiently $(<24 \mathrm{~h})$ responds to increased leaf water status (Munns, 2002). Therefore, rootderived chemical signals limit leaf growth in dry or saline soil. 
Because plant meristems are actively growing tissues where cell division governs sink strength, environmental signals can modulate plant responses to the growing conditions through changes in phytohormone concentrations, thus controlling assimilate partitioning between different sink tissues (Hartig and Beck, 2006). These hormonal changes not only influence the adaptive response but also affect the normal growth of the harvestable organs and thus influence economic productivity. Hence, plant hormones are considered a primary component of the signalling pathways controlling these processes.

This integrated plasticity in plant development probably involves long-distance communication between different organs with hormones, particularly differential changes in root and shoot hormone concentrations, playing an essential role (Sachs, 2005). Abscisic acid (ABA) is likely involved in this communication as it is found in xylem sap, and its xylem concentration increases after drought and salinity stress (Munns and Cramer, 1996). However, its role in growth regulation has been equivocal as different studies have suggested it can directly inhibit leaf elongation (Dodd and Davies, 1994), maintain it by restricting the evolution of ethylene, another potential growth inhibitor (Sharp and LeNoble, 2002) or have no effect (Martin-Vertedor and Dodd, 2011). Moreover, the origin of the ABA in the xylem sap is not known, for it moves readily in the phloem and recirculates from leaves to roots (Munns and Cramer, 1996). Thus, it seems that other hormones are involved in growth regulation of plants in drying or saline soil (reviewed by Dodd, 2005; Ghanem et al., 2011b; Pérez-Alfocea et al., 2010). Both cytokinins and auxins act as endogenous mitogens whose concentrations can be environmentally modulated to regulate the formation of roots and shoots and their relative sink strength.
Many of the relatively scarce studies conducted on hormonal root-to-shoot signaling measured only one or two of the major hormone groups following soil drying or a stepchange in salinity. Nevertheless, all plant species investigated seem to show conserved behavior with respect to increased xylem ABA concentration and decreased xylem cytokinin concentrations in response to drought or salt (Fig. 1). Explanation of stress-induced changes in biomass allocation by specific authors has generally favored the hormones that were measured, thus there are several divergent hypotheses that seemingly coexist in the literature (Munns and Cramer, 1996; Sachs, 2005; van der Werf and Nagel, 1996). The recent advent of simultaneous multianalyte techniques for hormone quantification allows a far more comprehensive analysis of the changes in plant hormone status following salinity and drought (Albacete et al., 2009).

\section{Abscisic Acid}

Typically, xylem ABA concentration increases much earlier and to a greater extent than bulk leaf ABA concentration during soil drying, and this increase is correlated with decreased stomatal conductance $\left(g_{s}\right)$ in a range of species (reviewed in Dodd, 2005; see also Fig. 1). Further evidence that long-distance transport of ABA was responsible for stomatal closure was provided by experiments demonstrating that soil application (Zhang and Davies, 1990) or stem injection (Tardieu et al., 1996) of ABA to well-watered plants generated a similar relationship between xylem ABA concentration and $g_{s}$ to that found in water-stressed plants. Similarly, removal of ABA from maize (Zea mays) xylem sap using an immunoaffinity column eliminated its antitranspirant activity,

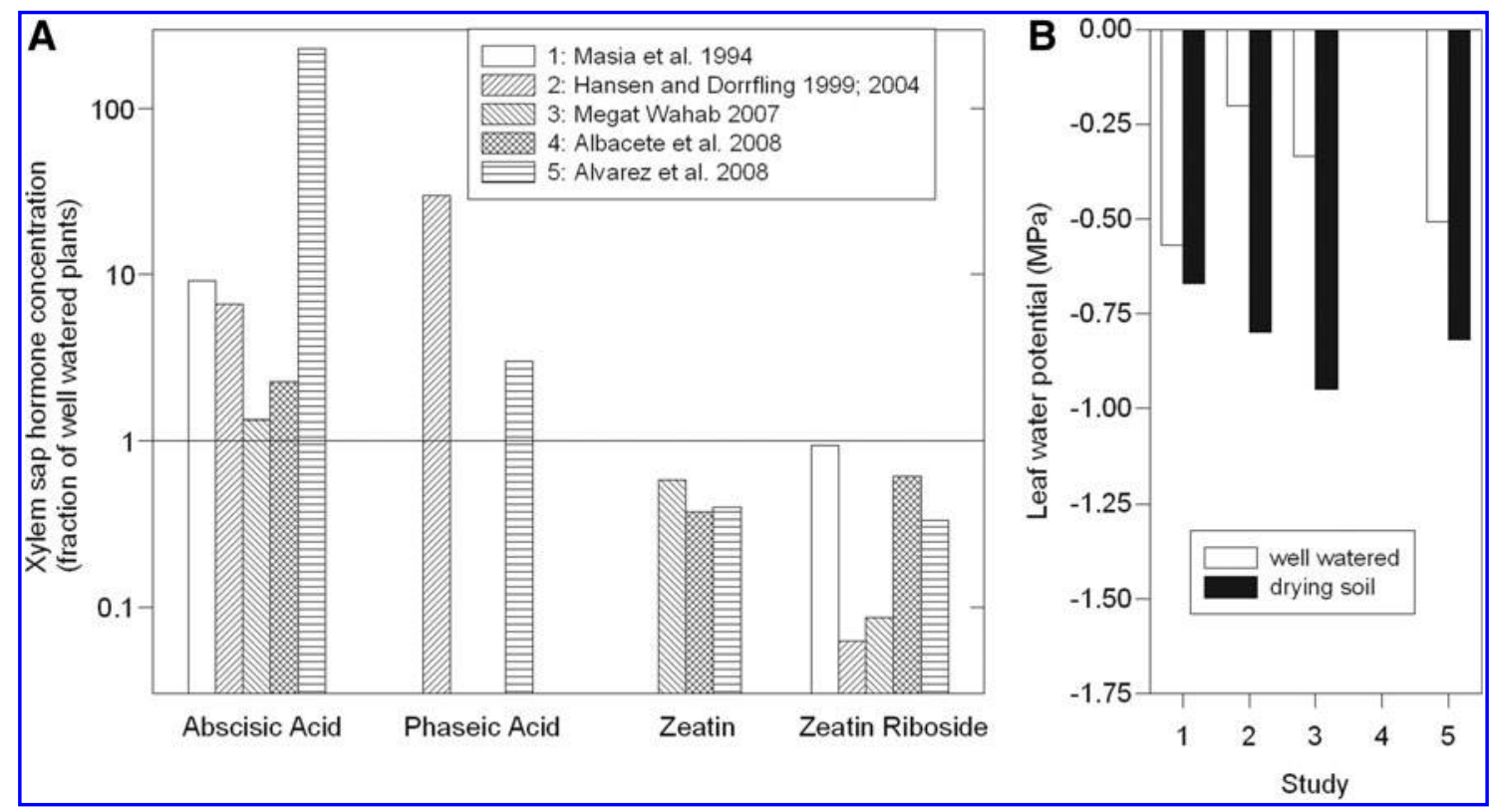

FIG. 1. (A) Fold change in xylem hormone concentration for sunflower (Hansen and Dorrfling, 1999, 2004; Masia et al., 1994), tomato (Albacete et al., 2008; Megat Wahab, 2007), and maize (Alvarez et al. 2008) in response to $100 \mathrm{mM} \mathrm{NaCl}$ for 15 days (Albacete et al., 2008) or soil drying (other studies). (B) Leaf water potential of plants grown in well watered (hollow bars) and drying (filled bars) soil in these studies. Xylem sap was collected applying pressure to the root system and collecting sap from the cut leaf surface of an otherwise intact plant (Megat Wahab, 2007), or from the root system of detopped plants by applying pressure to the roots (other studies). Absence of bars indicates that selected measurements were not made. 
as assessed using a detached leaf transpiration assay (Zhang and Davies, 1991).

However, several studies indicate that variation in xylem ABA concentration alone cannot always explain the extent of water stress-induced stomatal closure. In some species, the ABA concentration detected in xylem sap is apparently too low to influence stomatal conductance when xylem-supplied to detached leaves (e.g., Prunus armeniaca) (Loveys et al., 1987). Similarly, the antitranspirant activity of wheat (Triticum aestivum) xylem sap could not be explained in terms of its ABA concentration as assessed using a detached leaf transpiration assay, and removal of ABA from this xylem sap using an immunoaffinity column did not eliminate its antitranspirant activity (Munns and King, 1988).

Further work with grafted plants (a surgical technique to attach genetically different roots and shoots), while confirming the importance of xylem ABA concentration in regulating stomatal closure, has cast doubt on whether the root system is the principal source of the additional ABA detected as the soil dries or becomes saline. The role of root synthesized ABA in regulating stomatal and growth responses has been evaluated by reciprocal grafting of wild-type (WT), ABA-deficient, and more recently ABA overproducing genotypes, especially using the notabilis (not) and flacca (flc) ABA-deficient tomato mutants. In both WT self-grafts and WT/flc grafts, WT scions showed similar stomatal closure in response to drying soil (Holbrook et al., 2002), stomatal conductance, and shoot growth in well-watered soil when grown under low evaporative demand in a controlled environment room (Dodd et al., 2009) and shoot growth under control and saline $(75 \mathrm{mM}$ $\mathrm{NaCl}$ ) conditions when grown hydroponically (Chen et al., 2003). In contrast, leaf area and xylem sap ABA concentration of flc/WT (scion/rootstock) plants increased 1.6-fold and threefold compared to flc self-grafts, respectively, while xylem sap ACC concentration and leaf ethylene evolution was decreased to WT levels (Dodd et al., 2009). Although these data suggest little impact of the root system on shoot physiology except in the case of ABA-deficient scions, WT/flc plants had a lower water potential under higher evaporative demand (Ghanem et al., 2011b) presumably due to lower root hydraulic conductivity (Chen et al., 2003). Consequently, further evaluation of the role of root-supplied ABA from different rootstocks on shoot physiology, especially under dry or saline soil, seems warranted.

Because salt or drought stress can increase xylem ABA concentrations by several orders of magnitude (Fig. 1), there has also been interest in whether ABA conjugates or metabolites can affect shoot physiology. Although phaseic acid (the metabolic product of 8-OH hydroxylation of ABA) can also be detected in xylem sap if leaf water deficits occur (Fig. 1), it exerts a negligible antitranspirant activity (Sharkey and Raschke, 1982). ABA glucose ester (ABA-GE) occurs in the xylem sap of several plants (reviewed in Sauter et al., 2002) and the intercellular washing fluid of barley primary leaves contains a $\beta$-glucosidase activity (Dietz et al., 2000) that releases ABA from ABA-GE in the leaf apoplast. The activity of these $\beta$-glucosidases increased with salt stress (Dietz et al., 2000) so that xylem-transported ABA-GE is probably a source of stress-induced apoplastic ABA in the leaf. Apoplastic alkalinization, in response to local atmospheric conditions (e.g., high evaporative demand) or soil drying-induced changes in xylem sap $\mathrm{pH}$, will result in the release of chloroplastic ABA to the apoplast (Wilkinson and Davies, 2008; Wilkinson et al., 1998) and can initiate stomatal closure prior to any increase in xylem ABA concentration.

\section{Aminocyclopropane Carboxylic Acid (ACC)}

In response to soil flooding, tomato plants increased $x y-$ lem ACC (the immediate precursor of ethylene) delivery prior to the classical physiological and morphological responses of petiole ethylene production and epinastic curvature (Bradford and Yang, 1980). Supplying xylem ACC concentrations typically found in xylem sap of flooded tomato plants (at least $3 \mu \mathrm{M}$ ) to shoots detached from welldrained plants induced petiole ethylene evolution and epinasty (Bradford and Yang, 1980), suggesting that rootsourced ACC was responsible for shoot responses. However, the role of root-sourced ACC as a signal of saline or drying soil is more equivocal.

Salt shock ( $200 \mathrm{mM} \mathrm{NaCl}$ ) increased (threefold after 9 days) xylem ACC concentrations in hybrid citrange (Citrus sinensis $\times$ Poncirus trifoliata) (Gomez-Cadenas et al., 1998). Similarly, tomato plants required salinization (at $100 \mathrm{mM}$ $\mathrm{NaCl}$ ) for 15 days before xylem ACC concentration increased (Albacete et al., 2008), with further work demonstrating that the rootstock could double the xylem ACC concentrations detected (Albacete et al., 2009). Because ACC is likely phloemmobile (because it is a weak acid similar to ABA) and recirculates from leaves to roots, further work is required to determine whether these differences are due to intrinsic differences in root ACC synthesis, or due to differences in shoot ionic relations mediating ACC transport to the roots.

Water stress induction by transplanting bare-rooted mandarin seedlings to dry sand for $24 \mathrm{~h}$, which decreased leaf water potential to $-3.0 \mathrm{MPa}$, increased xylem ACC concentration (Tudela and Primo-Millo, 1992). A more moderate soil drying treatment of pea (Pisum sativum) which imposed a relatively stable soil water status (that did not exceed a matric potential of $-0.25 \mathrm{MPa}$ ) also roughly doubled xylem ACC concentration (Belimov et al., 2009). Nevertheless, additional work is necessary to establish whether increased xylem ACC concentration is a typical response to soil drying, especially because the prevailing view in the literature that soil drying does not enhance leaf ethylene evolution (Morgan et al., 1990).

\section{Cytokinins}

It has been hypothesized decreased CK supply from the root to the shoot could inhibit leaf growth while a low CK content would promote root growth and thus the root/shoot ratio (Rahayu et al., 2005; van der Werf and Nagel, 1996). Because water and salt stress decrease CK concentration in xylem sap (Fig. 1), correlative and functional studies have reported a close relationship between decreased bioactive $\mathrm{CK}$ concentrations in the xylem sap and increased leaf senescence and decreased plant growth and productivity under water (Rivero et al., 2007) and saline (Albacete et al., 2009, 2010; Ghanem et al., 2008, 2011a) stresses. Although some cytokinin species (e.g., BAP) actually show increased concentrations in response to soil drying (Alvarez et al., 2008), it is difficult to drawn solid conclusions about the role of this hormone class in root-to-shoot signaling due to the complexity of CK metabolism. 
Under salt stress, both leaf growth and delayed senescence were positively correlated with rootstock-mediated zeatin $(Z)$ and $\mathrm{K}^{+}$concentrations in the leaf xylem sap and also with hormonal ratios between $\mathrm{CKs}$ and $\mathrm{ACC}$ (Z/ACC and Z + $\mathrm{ZR} / \mathrm{ACC}$ ), whereas the ratio ACC/ABA was negatively correlated with leaf biomass (Ghanem et al., 2008). It was suggested that early hormonal signals coming from the roots positively (CKs, $\mathrm{ABA}$ ) or negatively (ACC) influenced leaf growth, while lower xylem CK concentrations or higher ACC concentrations promoted leaf senescence (Pérez-Alfocea et al., 2010), and the balance of different compounds may be crucial in regulating physiological responses. Maintaining growth while minimizing senescence may provide more energy to maintain ionic homeostasis by acting on both root ion uptake $(\mathrm{K}+)$ and efflux $(\mathrm{Na}+)$ and by diluting toxic ions through growth (Pérez-Alfocea et al., 2010). Although the bioactive cytokinins ( $\mathrm{tZ}, \mathrm{tZR}, \mathrm{IP}, \mathrm{IPR})$ seem key in regulating growth and senescence processes as described above, the role of other CK groups that significantly increase under stress conditions such as cis-CKs should be investigated (Ghanem et al., 2011a).

It has been suggested that the ABA:cytokinin ratios in xylem sap are important for stress signalling (Hansen and Dorffling, 1999). Overexpression of the isopentenyltransferase gene under the control of a heat-shock-inducible promoter (HSP70::ipt) in tomato plants roughly doubled the concentration of bioactive cytokinins ( $\mathrm{tZ}, \mathrm{tZR}$ ) in the xylem sap and decreased $\mathrm{ABA}$ by $30 \%$ under $100 \mathrm{mM} \mathrm{NaCl}$ stress (Ghanem et al., 2011a). The decreased ABA/CK concentration in the leaf xylem sap and leaf tissues was related with enhanced transpiration and growth and delayed leaf senescence, suggesting a close interaction between both groups of hormones. Similarly, it has also been suggested that the ABA/CKs ratio could be considered as a master regulator of nodule activity within Medicago ciliaris plants, and thus regulating positively (CK by nodules) and negatively (ABA by the plant) the symbiotic-parasitic relationship, especially under limiting growing conditions as salt stress (Ben-Salah et al., 2011).

\section{Auxins and Hydraulic Root-to-Shoot Signalling}

Although auxins have not been classically considered as xylem sap components, these hormones seem to have an important role in controlling xylem properties and, therefore, root hydraulic conductivity. Because auxin is a key component in the expansion zone of the developing xylem that regulates lumen area of tracheary elements (Zakrewski, 1991), stress-induced changes in vessel lumen area may be caused by decreases in auxin concentrations of the developing cambium. In poplar, diminished auxin content may be a factor that adapts growth and wood development during adverse environmental conditions (Popko et al., 2010) and 2 weeks of salt stress $(75 \mathrm{mM} \mathrm{NaCl})$ decreased the xylem differentiation zone and vessel lumen area in the salt-sensitive Populus $\times$ canescens (Escalante-Perez et al., 2009). Xylem sap proteome analysis of Brassica oleracea in response to salt stress demonstrated the accumulation of enzymes involved in xylem differentiation and lignification, such as cystein proteinases, acid peroxidases, and a putative hydroxycinnamoyl-CoA:shikimate hydroxycinnamoyl transferase (Fernández-García et al., 2011). These results suggest that xylem differentiation and lignification induced by salt stress could be considered as an adaptive response to increase resistance to xylem cavitation, although to the detriment of stem hydraulic conductivity, leaf photosynthesis, and therefore plant growth and productivity (Stiller, 2009). Additionally, overexpression of the AtGH3.5 gene decreased free auxin concentrations but increased concentrations of conjugated IAA-Asp, and decreased growth but enhanced stress resistance. Consequently, it was suggested that GH3 auxin conjugate synthases are involved in adjusting plant hormonal balance in response to stress and thus supporting the adaptive role described above (Park et al., 2007). Moreover, ABA stimulation of GH3 genes (Seo et al.,2009) may mediate ABA-auxin crosstalk affecting lateral root development.

\section{Ionomics of Drying and Saline Soil}

Soil drying alters the rate of diffusion of nutrients from the soil matrix to the root surface, root nutrient uptake, and xylem loading of plant nutrients. Thus, it is reasonable to question whether xylem sap ion concentrations could constitute a sensitive "early warning signal" of drying soil. In favor of this argument, as the soil dries, xylem sap ion concentrations (nitrate and ammonium) were decreased (compared to wellwatered plants) prior to any change in leaf nitrogen concentrations (e.g., Bahrun et al., 2002). Consequently, many studies have sought to characterize changes in xylem sap ion concentrations as the soil dries (summarized in Fig. 2). It is difficult to directly compare these studies due to differences in soil type and nutrient status, plant species, the degree of soil drying, and xylem sap sampling methodology. Nevertheless, three different xylem sampling methodologies (sap collected from the root system via root pressure alone, or via pressurization of the root system, or sap collected from intact leaves following root pressurization) gave similar relative ionic changes following a mild soil drying treatment that did not perturb maize leaf water potential (Goodger et al., 2005). However, both increases and decreases in the major ions (nitrate, phosphate, chloride, calcium, sodium, magnesium, and potassium) occurred (Fig 2). Decreases in sap flow rate due to stomatal closure might be expected to increase the concentration of all xylem sap constituents (assuming no change in the xylem loading rate). Indeed, all cations and anions sampled showed an exponential decline in xylem sap concentration as sap flow rate increased (Else et al., 1995; Schurr and Schulze, 1996). The decreased concentration of some ions following soil drying indicates decreases in xylem loading and/or uptake rate.

Of all the ions considered in studies of soil drying, xylem sulphate concentration either showed no change in response to a moderate soil drying treatment (Munns and King 1988), or was consistently increased following soil drying (Fig. 2). This increase was correlated with an increase in sulphate transporter genes in maize roots, and occurred prior to any increase in expression of ABA biosynthesis genes (Ernst et al., 2010). It is necessary to determine whether xylem sulphate concentrations always increases in response to drying soil, irrespective of actual bulk soil or rhizosphere sulphate concentrations. To examine the role of additional xylem-supplied sulphate in inducing stomatal closure mediated by drying soil, artificial xylem saps containing different concentrations of sulphate and ABA were supplied to detached maize leaves in a transpiration bioassay (Ernst et al., 2010). Although supplying $2 \mathrm{mM} \mathrm{MgSO}_{4}$ via the xylem had a small (not significant) effect 


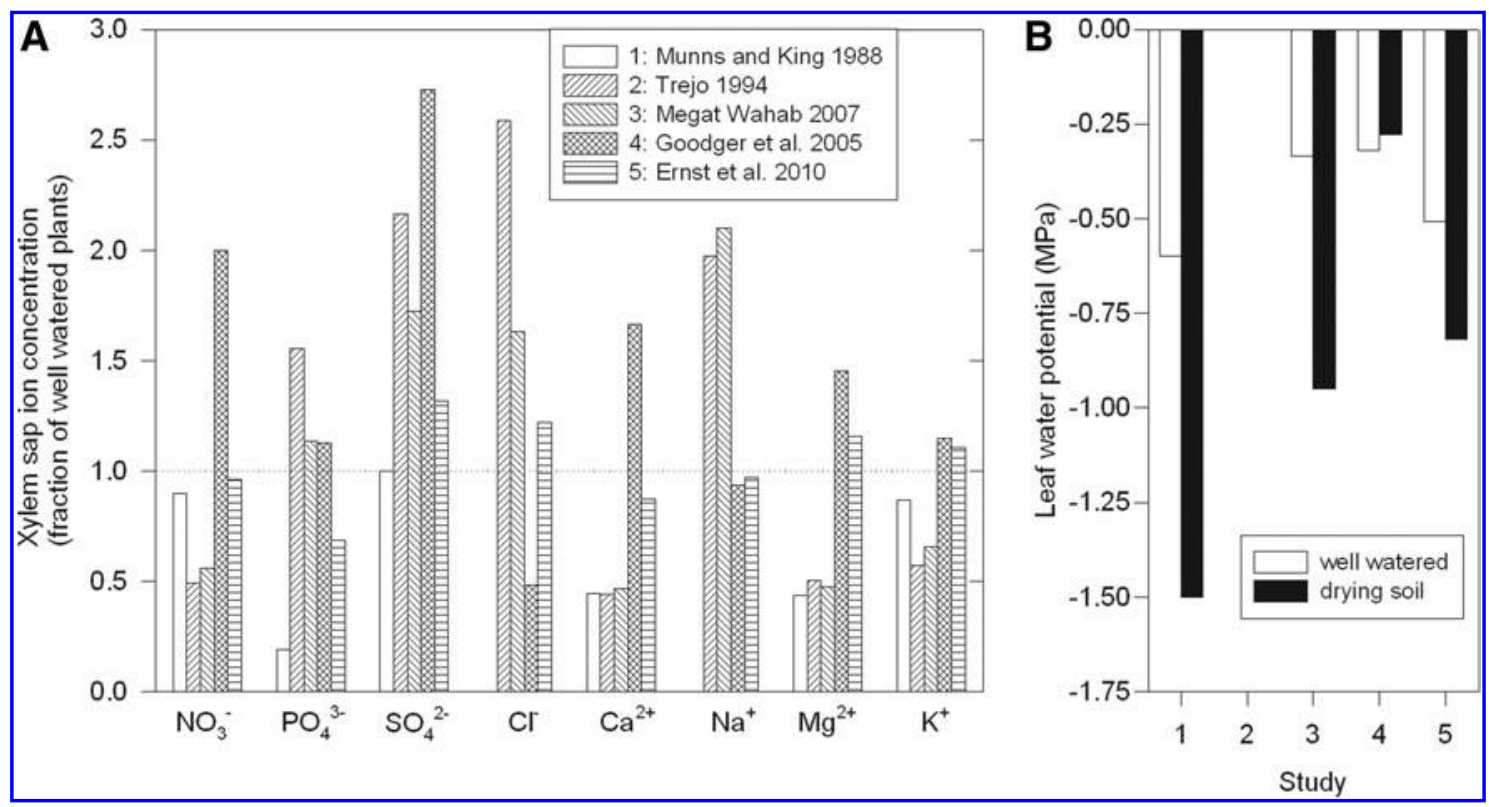

FIG. 2. (A) Fold change in xylem ion concentration for wheat (Munns and King, 1988), bean (Trejo, 1994), tomato (Megat Wahab, 2007), and maize (Ernst et al., 2010; Goodger et al.. 2005) in response to soil drying. (B) Leaf water potential of plants grown in well watered (hollow bars) and drying (filled bars) soil in these studies. Xylem sap was collected from the root system of detopped plants by applying pressure to the roots (Ernst et al., 2010; Goodger et al., 2005) and from the shoots by either pressurizing detached shoots in a Scholander-type pressure chamber (Trejo, 1994) or applying pressure to the root system and collecting sap from the cut leaf surface of an otherwise intact plant (Megat Wahab, 2007; Munns and King, 1988). Absence of bars indicates that selected measurements were not made.

on detached leaf transpiration in the absence of xylem supplied ABA, cosupply of $2 \mathrm{mM} \mathrm{MgSO}_{4}$ and $0.3 \mu \mathrm{M}$ ABA caused $20 \%$ more stomatal closure than that observed when $0.3 \mu \mathrm{M}$ ABA was supplied alone. A similar effect on transpiration was noted when a higher ABA concentration $(1 \mu \mathrm{M})$ was supplied to detached maize leaves, and supplying sulphate to the incubation medium of epidermal strips of Vicia faba sensitized stomatal closure to ABA. Further work is needed to evaluate the physiological significance of xylem-borne sulphate, especially since sulphate ions can often be a component of saline soils.

Xylem nitrate concentration also changed in response to soil drying (Fig. 2), with initial increases being followed by decreased nitrate concentration as the soil dried further (Wilkinson et al., 2007). Elevated xylem nitrate concentration ( $>10 \mathrm{mM}$ in sensitive genotypes) can exert a direct inhibitory effect on transpiration or leaf elongation of detached maize shoots, mediated by apoplastic alkalinization (Jia and Davies, 2007), which affects the partitioning of ABA between symplast and apoplast (Wilkinson et al., 1998, 2007). Analogous to the effect described for sulphate, high xylem sap nitrate concentrations also increased the sensitivity of detached leaves to xylem-supplied ABA. Thus, transpiration of detached maize leaves was inhibited by supplying different solutions via the xylem stream: by $5 \%$ for $20 \mathrm{mM} \mathrm{KNO}, 18 \%$ for $0.1 \mu \mathrm{M} \mathrm{ABA}$ and $25 \%$ by a combined treatment (Wilkinson et al., 2007). Interestingly, nitrate deprived cotton plants were also more sensitive to xylem-supplied ABA (Radin et al., 1982), again likely due to apoplastic alkalinization (Dodd et al., 2003) altering the partitioning of ABA between apoplast and symplast. Thus, optimal plant nutrition results in the least sensitive stomatal and leaf growth responses to ABA loaded into the transpiration stream, but sub- or supraoptimal xylem nitrate concentrations can sensitize plant responses (Wilkinson et al., 2007).

Changes in xylem ion concentration in response to salinity have almost exclusively focused on sodium and chloride ion concentrations. Nevertheless, a recent study indicates that a 7-day exposure of Brassica oleracea to $80 \mathrm{mM} \mathrm{NaCl}$ (FernandezGarcia et al., 2011) increased xylem sap potassium concentration (1.3-fold), calcium concentration (1.6-fold), and nitrate concentration (3.7-fold). Increases in potassium and phosphate concentrations were also reported in the xylem sap of salinized tomato plants, while $\mathrm{Ca}^{2+}$ and $\mathrm{Mg}^{2+}$ were not clearly affected after 3 weeks under $100 \mathrm{mM} \mathrm{NaCl}$ stress (Pérez-Alfocea et al., 2000). The causes (changes in nutrient uptake, xylem loading, or sap flow rate) and physiological significance of these changes requires further work. However, because salinity usually decreases transpiration rates (Munns, 2011), these changes in concentration may not actually increase delivery rates of these ions to the shoot, consistent with many observations of decreased tissue nutrient status in salinized plants (Hu et al., 2005). Furthermore, it should be noted that xylem sodium and chloride ion concentrations were increased by at least an order of magnitude more in tomato and oilseed rape (Fernandez-Garcia et al., 2011; PérezAlfocea et al., 2000).

\section{Proteomics of Drying and Saline Soil}

Although the existence of proteins in xylem sap has been known for at least two decades (Biles and Abeles, 1991), only recently have researchers begun to investigate the qualitative and quantitative changes caused by dry or saline soil. 
Although soil drying or salinization increased total xylem sap protein composition and induced qualitative changes in protein composition as assessed by SDS-PAGE in a range of species (Zhu and Zhang, 1997), increasing the protein concentration fed to detached leaves had no antitranspirant effect, nor did it enhance the antitranspirant effect of ABA. Irrespective of sap source and protein concentration, there was a unifying relationship between sap ABA concentration and the growth inhibitory or antitranspirant properties of the sap. More recently, a comprehensive proteomic study (Alvarez et al., 2008) identified 39 xylem sap proteins in maize that were differentially expressed in response to soil drying. Proteins whose abundance changed were assigned to two biological categories: those involved in cell wall metabolism (e.g., peroxidase, xyloglucan endotransglycosylase, polygalacturonase inhibitor and pectin methylesterase) and those putatively involved in plant defence (e.g., thaumatin-like pathogenesis-related protein, zeatin-like protein, cupin family protein, putative germin A, class IV chitinase and b-1,3glucanase) although some proteins (e.g., peroxidases) may be involved in both functions. The increased abundance of cationic peroxidases in xylem sap, along with increases in phenylpropanoids was postulated to lead to decreased lignin biosynthesis in xylem vessels and induce cell wall stiffening (Alvarez et al., 2008), thereby increasing the resistance of xylem vessels to the increased xylem tensions that accompany water deficits. Further proteomic studies of xylem sap composition seem warranted, especially because the role of these changes has yet to be elucidated.

\section{Conclusions}

Of the Omics measurements made on xylem sap, most attention has been given to the role of ionomics in mediating responses to salt stress, and the role of xylem hormonal status in mediating responses to drying soil. Increasingly, it is appreciated that interactions between ion and hormonal status are important in regulating physiological responses. Manipulation of xylem sap composition (e.g., by grafting plants on selected rootstocks) has produced positive effects on growth and yield under suboptimal (resource limiting) conditions (Albacete et al., 2009; Ghanem et al., 2011a), with no penalties under optimal (nonresource limiting) conditions. Although root-sourced CKs, ABA, and ACC seem key in controlling shoot performance under abiotic stress, further insights about their physiological and genetic determinants and their regulation will certainly facilitate rootstock genetic improvement (Asins et al., 2010). Rootstock hormone status may also be important in determining tolerance to biotic stress, another highly desirable agronomic trait (Ghanem et al., 2011b).

\section{Acknowledgments}

The authors thank BBSRC and DEFRA (UK), Fundación Séneca de la Región de Murcia (project 08712/PI/08) and MICINN-FEDER (project AGL2008-01733/AGR) (Spain), and the European Commission for supporting ongoing research on root-to-shoot signalling (ROOTOPOWER Project 289365).

\section{Author Disclosure Statement}

The authors declare that no conflicting financial interests exist.

\section{References}

Albacete, A., Ghanem, M.E., Martinez-Andujar, C., Acosta, M., Sanchez-Bravo, J., Martinez, V., et al. (2008). Hormonal changes in relation to biomass partitioning and shoot growth impairment in salinised tomato (Solanum lycopersicum L.) plants. I Exp Botany 59, 4119-4131.

Albacete, A., Martinez-Andujar, C., Ghanem, M.E., Acosta, M., Sanchez-Bravo, J., Asins, M.J., et al. (2009). Rootstockmediated changes in xylem ionic and hormonal status are correlated with delayed leaf senescence and increased leaf area and crop productivity in salinised tomato. Plant Cell Environ 32, 928-938.

Alvarez, S., Marsh, E.L., Schroeder, S.G., and Schactman, D.P. (2008). Metabolomic and proteomic changes in the xylem sap of maize under drought. Plant Cell Environ 31, 325-340.

Arbona, V., Argamasilla, R., and Gómez-Cadenas, A. (2010). Common and divergent physiological, hormonal and metabolic responses of Arabidopsis thaliana and Thellungiella halophila to water and salt stress. I Plant Physiol 167, 13421350.

Asins, M.J., Bolarin, M.C., Pérez-Alfocea, F., Estan, M.T., Martínez-Andújar, C., Albacete, A., et al. (2010). Genetic analysis of physiological components of salt tolerance conferred by Solanum rootstocks. What is the rootstock doing for the scion? Theoret Appl Genet 121, 105-115.

Bahrun, A., Jensen, C.R., Asch, F., and Mogensen, V.O. (2002). Drought induced changes in xylem $\mathrm{pH}$, ionic composition, and ABA concentration act as early signals in field-grown maize (Zea mays L.). J Exp Botany 53, 251-263.

Belimov, A.A., Dodd, I.C., Hontzeas, N., Theobald, J.C., Safronova, V., and Davies, W.J. (2009). Rhizosphere bacteria containing ACC deaminase increase yield of plants grown in drying soil via both local and systemic hormone signalling. New Phytol 181, 413-423.

Ben Salah, I., Albacete, A., Messedi, D., Gandour, M., Martínez Andújar, C., Zribi, K., et al. (2011). Hormonal responses of nodulated Medicago ciliaris lines differing in salt tolerance. Environ Exp Botany. doi: 10.1016/j.envexpbot.2011.04.013

Biles, C.L., and Abeles, F.B. (1991). Xylem sap proteins. $\underline{\text { Plant }}$ Physiol 96, 597-601.

Bohnert, H.J., Gong, Q.Q., Li, P.H., and Ma, S.S. (2006). Unraveling abiotic stress tolerance mechanisms-getting genomics going. Curr Opin Plant Biol 9, 180-188.

Bradford, K.J., and Yang, S.F. (1980). Xylem transport of 1-aminocyclopropane-1-carboxylic acid, an ethylene precursor, in waterlogged tomato plants. Plant Physiol 65, 322-326.

Charlton, A.J., Donarski, J.A., Harrison, M., Jones, S.A., Godward, J., Oehlschlager, S., et al. (2008). Responses of the pea (Pisum sativum L.) leaf metabolome to drought stress assessed by nuclear magnetic resonance spectroscopy. Metabolomics 4 , 312-327.

Chen, G., Fu, X., Lips, H., and Sagi, M. (2003). Control of plant growth resides in the shoot, and not in the root, in reciprocal grafts of flacca and wild-type tomato (Lysopersicon esculentum), in the presence and absence of salinity stress. Plant Soil 256, 205-215.

De Vos, R.C., Moco, S., Lommen, A., Keurentjes, J.J., Bino, R.J., and Hall, R.D. (2007). Untargeted large-scale plant metabolomics using liquid chromatography coupled to mass spectrometry. Nat Protocols 2, 778-791.

Dietz, K.J., Wichert, K., Sauter, A., Messdaghi, D., and Hartung, W. (2000). Characterisation of an extracellular B-glucosidase in barley involved in the hydrolysis of ABA glucose conjugate in leaves. J Exp Botany 51, 937-944. 
Dodd, I.C. (2005). Root-to-shoot signalling: assessing the roles of "up" in the up and down world of long-distance signalling in planta. Plant Soil 274, 257-275.

Dodd, I.C., and Davies, W.J. (1994). Leaf growth responses to ABA are temperature dependent. J Exp Botany 45, 903-907.

Dodd, I.C., Tan, L.P., and He, J. (2003). Do increases in xylem sap $\mathrm{pH}$ and/or ABA concentration mediate stomatal closure following nitrate deprivation ? J Exp Botany 54, 1281-1288.

Dodd, I.C., Theobald, J.C., Richer, S.K., and Davies, W.J. (2009). Partial phenotypic reversion of ABA-deficient flacca tomato (Solanum lycopersicum) scions by a wild-type rootstock: normalizing shoot ethylene relations promotes leaf area but does not diminish whole plant transpiration rate. J Exp Botany 60 4029-4039.

Dodd, I.C., Egea, G., Watts, C., and Whalley, W.R. (2010). Root water potential integrates discrete soil physical properties to influence ABA signalling during partial rootzone drying. I Exp Botany 61, 3543-3551.

Else, M.A., Hall, K.C., Arnold, G.M., Davies, W.J., and Jackson, M.B. (1995). Export of abscisic acid, 1-aminocyclopropane-1carboxylic acid, phosphate, and nitrate from roots to shoots of flooded tomato plants-accounting for effects of xylem sap flow rate on concentration and delivery. Plant Physiol 107, 377-384.

Enot, D.P., Beckman, M., and Draper, J. (2007). Detecting a difference-assessing generalisability when modelling metabolome fingerprint data in longer term studies of genetically modified plants. Metabolomics 3, 335-347.

Ernst, L., Goodger, J.Q.D., Alvarez, S., Marsh, E.L., Berla, B., Lockhart, E., et al. (2010). Sulphate as a xylem-borne chemical signal precedes the expression of ABA biosynthetic genes in maize roots. J Exp Botany 61, 3395-3405.

Escalante-Perez, M., Lautner, S., Nehls, U., Selle, A., Teuber, M., Schnitzler, J.P., et al. (2009). Salt stress affects xylem differentiation of grey poplar (Populus $x$ canescens). Planta 229, 299-309.

Fernandez-Garcia, N., Hernandez, M., Casado-Vela, J., Bru, R., Elortza, F., Hedden, P., et al. (2011). Changes to the proteome and targeted metabolites of xylem sap in Brassica oleracea in response to salt stress. Plant Cell Environ 34, 821-834.

Fiehn, O., Kopka, J., Dormann, P., Altmann, T., Trethewey, R.N., and Willmitzer, L. (2000). Metabolite profiling for plant functional genomics. Nat Biotechnol 18, 1157-1161.

Gagneul, D., Ainouche, A., Duhaze, C., Lugan, R., Lahrer, F.R., and Bouchereau, A. (2007). A reassessment of the function of the so-called compatible solutes in the halophytic Plumbaginaceae Limonium latifolium. Plant Physiol 144, 1598-1611.

Ghanem, M.E., Albacete, A., Martinez-Andujar, C., Acosta, M., Romero-Aranda, R., Dodd, I.C., et al. (2008). Hormonal changes during salinity-induced leaf senescence in tomato (Solanum lycopersicum L.). J Exp Botany 59, 3039-3050.

Ghanem, M.E., Albacete, A., Smigocki, A.C., Frebort, I., Pospíšilová, H., Martínez-Andújar, C., et al. (2011a). Rootsynthesised cytokinins improve shoot growth and fruit yield in salinised tomato (Solanum lycopersicum L.). I Exp Botany 62, 125-140.

Ghanem, M.E., Hichri, I., Smigocki, A.C., Albacete, A., Fauconnier, M.L., Diatloff, E., et al. (2011b). Root-targeted biotechnology to mediate hormonal signalling and improve crop stress tolerance. Plant Cell Rep 30, 807-823.

Gomez-Cadenas, A., Tadeo, F,R., Primo-Millo, E., and Talon, M. (1998). Involvement of abscisic acid and ethylene in the responses of citrus seedlings to salt shock. Physiol Plant 103, 475-484.
Gong, Q., Li, P., Ma, S., Indu Rupassara, S., and Bohnert, H.J. (2005). Salinity stress adaptation competence in the extremophile Thellungiella halophila in comparison with its relative Arabidopsis thaliana. Plant J 44, 826-839.

Goodger, J.Q.D., Sharp, R.E., Marsh, E.L., and Schactman, D.P. (2005). Relationships between xylem sap constituents and leaf conductance of well-watered and water-stressed maize across three xylem sap sampling techniques. I Exp Botany 56, 2389-2400.

Hansen, H., and Dorffling, K. (1999). Changes of free and conjugated abscisic acid and phaseic acid in xylem sap of drought-stressed sunflower plants. I Exp Botany 50, 15991605.

Hartig, K., and Beck, E. (2006). Crosstalk between auxin, cytokinins, and sugars in the plant cell cycle. Plant Biol 8, 389-396.

Holbrook, N.M., Shashidhar, V.R., James, R.A., and Munns, R. (2002). Stomatal control in tomato with ABA-deficient roots: response of grafted plants to soil drying. I Exp Botany 53, 1503-1514.

$\mathrm{Hu}$, Y.C., Fricke, W., and Schmidhalter, U. (2005). Salinity and the growth of non-halophytic grass leaves: the role of mineral nutrient distribution. Funct Plant Biol 32, 973-985.

Jia, W., and Davies, W.J. (2007). Modification of leaf apoplastic $\mathrm{pH}$ in relation to stomatal sensitivity to root-sourced ABA signals. Plant Physiol 143, 68-77.

Kim, J.K., Bamba, T., Harada, K., Fukusaki, E., and Kobayashi, A. (2007). Time-course metabolic profiling in Arabidopsis thaliana cell cultures after salt stress treatment. J Exp Botany 58, 415-424.

Loveys, B.R., Robinson, S., and Downton, W.J.S. (1987). Seasonal and diurnal changes in abscisic acid and water relations of apricot leaves (Prunus armeniaca L.). New Phytol 107, 15-27.

Martínez-Andujar, C. (2006). Análisis funcional de una asparragina sintetasa dependiente de amonio en tomate (Solanum lycopersicum L.) cultivado en condiciones de salinidad y diferente nutrición nitrogenada. $\mathrm{PhD}$ Thesis. Universidad de Murcia.

Martin-Vertedor, A.I., and Dodd, I.C. (2011). Root-to-shoot signalling when soil moisture is heterogeneous: increasing the proportion of root biomass in drying soil inhibits leaf growth and increases leaf ABA concentration. Plant Cell Environ 34, 1164-1175.

Megat Wahab, P.E. (2007). Soil drying effects on two tomato cultivars. PhD thesis. University of Lancaster.

Morgan, P.W., He, C.J., De Greef, J.A., and De Proft, M.P. (1990). Does water deficit stress promote ethylene synthesis by intact plants? Plant Physiol 94, 1616-1624.

Munns, R. (2002). Salinity, growth and phytohormones. In Salinity: environment-plants-molecules. A. Läuchli, and U. Lüttge, eds. (Kluwer Academic Publishers, New York), pp. 271-290.

Munns, R. (2011). Plant adaptations to salt and water stress: differences and commonalities. Adv Botanical Res 57, 1-32.

Munns, R., and Cramer, G.R. (1996). Is coordination of leaf and root growth mediated by abscisic acid? Opinion. Plant Soil $185,33-49$.

Munns, R., and King, R.W. (1988). Abscisic acid is not the only inhibitor of transpiration in xylem sap of wheat plants. Plant Physiol 88, 703-708.

Park, J.-E., Park, J.-Y., Kim, Y.-S., Staswick, P.E., Jeon, J., Yun, J., et al. (2007). GH3-mediated auxin homeostasis links growth regulation with stress adaptation response in Arabidopsis. J Biol Chem 282, 10036-10046.

Pérez-Alfocea, F., Balibrea, M.E., Alarcón, J.J., and Bolarín, M.C. (2000). Composition of phloem and xylem exudates in relation 
to the salt-tolerance of domestic and wild tomato species. J Plant Physiol 156, 367-374.

Pérez-Alfocea, F., Albacete, A., Ghanem, M.E., and Dodd, I.C. (2010). Hormonal regulation of source-sink relations to maintain crop productivity under salinity: a case study of root-toshoot signalling in tomato. Funct Plant Biol 37, 592-603.

Pfautsch, S., Gessler, A., Adams, M.A., and Rennenberg, H. (2009). Using amino-nitrogen pools and fluxes to identify contributions of understory Acacia spp. to overstorey Eucalyptus regnans and stand nitrogen uptake in temperate Australia. New Phytol 183, 1097-1113.

Popko, J., Hänsch, R., Mendel, R.-R., Polle, A., and Teichmann, T. (2010). The role of abscisic acid and auxin in the response of poplar to abiotic stress. Plant Biol 12, 242-258.

Radin, J.W., Parker, L.L., and Guinn, G. (1982). Water relation of cotton plants under nitrogen deficiency. V. Environmental control of abscisic acid accumulation and stomatal sensitivity to abscisic acid. Plant Physiol 70, 1066-1070.

Rahayu, Y.S., Walch-Liu, P., Neumann, G., von Wirén, N., and Bangerth, F. (2005). Root-derived cytokinins as long-distance signals for $\mathrm{NO}_{3}{ }^{-}$-induced stimulation of leaf growth. I Exp Botany 56, 1143-1152.

Rivero, R.M., Shulaev, V., and Blumwald, E. (2009). Cytokinindependent photorespiration and the protection of photosynthesis during water deficit. Plant Physiol 150, 1530-1540.

Ruan, C.J., and Teixeira da Silva, J.A. (2010). Metabolomics: creating new potentials for unraveling the mechanisms in response to salt and drought stress and for the biotechnological improvement of xero-halophytes. Crit Rev Biotechnol X, 1-17.

Sachs, T. (2005). Auxin's role as an example of the mechanisms of shoot/root relations. Plant Soil 268, 13-19.

Sauter, A.., Dietz, K.-J., and Hartung, W. (2002). A possible stress physiological role of abscisic acid conjugates in root-to-shoot signalling. Plant Cell Environ 25, 223-228.

Schachtman, D.P., and Goodger, J.Q.D. (2008). Chemical root to shoot signaling under drought Trends Plant Sci 13, 281-287.

Schurr, U., and Schulze, E.-D. (1996). Effects of drought on nutrient and ABA transport in Ricinus communis. Plant Cell Environ 19, 665-674.

Seo, P.D., Xiang, F., Qiao, M., Park, J.-Y., Lee, Y.N., Kim, S.-G., et al. (2009). The MYB96 transcription factor mediates abscisic acid signaling during drought stress response in Arabidopsis. Plant Physiol 151, 275-289.

Sharkey, T.D., and Raschke, K. (1982). Effects of phaseic acid and dihydrophaseic acid on stomata and the photosynthetic apparatus. Plant Physiol 65, 291-297.

Sharp, R.E., and LeNoble, M.E. (2002). ABA, ethylene and the control of root and shoot growth under water stress. J Exp Botany 53, 33-37.

Stiller, V. (2009). Soil salinity and drought alter wood density and vulnerability to xylem cavitation of baldcypress (Taxodium distichum (L.) Rich.) seedlings. Environ Exp Botany 67, 164-171.

Tardieu, F., Lafarge, T., and Simonneau, T. (1996). Stomatal control by fed or endogenous xylem ABA in sunflower: interpretation of correlations between leaf water potential and stomatal conductance in anisohydric species. Plant Cell Environ 19, 75-84.

Trejo, C.L. (1994). Involvement of abscisic acid in the regulation of stomatal aperture during soil drying. PhD thesis. University of Lancaster.

Tudela. D., and Primo-Millo, E. (1992). 1-Aminocyclopropane-1carboxylic acid transported from roots to shoots promotes leaf abscission in cleopatra mandarine (Citrus reshni Hort ex Tan) seedlings rehydrated after water-stress. Plant Physiol 100, 131-137.

van der Werf, A., and Nagel, O.W. (1996). Carbon allocation to shoots and roots in relation to nitrogen supply is mediated by cytokinins and sucrose: opinion. Plant Soil 185, 21-32.

Wilkinson, S., and Davies, W.J.. (2008). Manipulation of the apoplastic $\mathrm{pH}$ of intact plants mimics stomatal and growth responses to water availability and microclimatic variation. J Exp Botany 59, 619-631.

Wilkinson, S., Bacon, M.A., and Davies, W.J. (2007). Nitrate signalling to stomata and growing leaves: interactions with soil drying, ABA, and xylem sap $\mathrm{pH}$ in maize. I Exp Botany 58, 1705-1716.

Wilkinson, S., Corlett, J.E., Oger, L., and Davies, W.J. (1998). Effects of xylem $\mathrm{pH}$ on transpiration from wild-type and flacca tomato leaves: a vital role for abscisic acid in preventing excessive water loss even in well-watered plants. Plant Physiol 117, 503-509.

Zakrewski, J. (1991). Effect of indole-3-acetic acid (IAA) and sucrose on vessel size and density in isolated stem segments of oak (Quercus robur). Physiol Plant 81, 234-238.

Zhang, J., and Davies, W.J. (1990). Does ABA in the xylem control the rate of leaf growth in soil-dried maize and sunflower plants? J Exp Botany 41, 1125-1132.

Zhang, J., and Davies, W.J. (1991). Antitranspirant activity in xylem sap of maize plants. J Exp Botany 42, 317-321.

Zhu, X.H., and Zhang, J.H. (1997). Anti-transpiration and antigrowth activities in the xylem sap from plants under different types of soil stress. New Phytol 137, 657-664.

Zuther, E., Koehl, K., and Kopka. (2007). Comparative metabolome analysis of the salt response in breeding cultivars of rice. In Advances in Molecular Breeding Toward Drought and Salt Tolerance Crops. M.A. Jenks, P.M. Hasegawa, and S.M. Jain, eds. (Springer-Verlag, Berlin), pp. 285-315.

Address correspondence to: Francisco Pérez-Alfocea Departamento de Nutrición Vegetal Centro de Edafología y Biología Aplicada del Segura (CEBAS) Consejo Superior de Investigaciones Científicas (CSIC) Campus Universitario de Espinardo P.O. Box, 164 E-30100, Espinardo, Murcia, Spain.

E-mail: alfocea@cebas.csic.es 
